\title{
Integrative Structure and Functional Anatomy of a Nuclear Pore Complex
}

Nudelman I. ${ }^{1}$, Kim S.J. ${ }^{2}$, Fernandez-Martinez J. ${ }^{1}$, Shi Y. ${ }^{3}$, Zhang W. ${ }^{3}$, Raveh B. ${ }^{2}$, Herricks T. ${ }^{4}$, Slaughter B.D. ${ }^{5}$, Hogan J. ${ }^{6}$, Upla P. ${ }^{7}$, Chemmama I.E. ${ }^{2}$, Pellarin R. ${ }^{2}$, Echeverria I. ${ }^{2}$, Shivaraju M. ${ }^{5}$, Chaudhury A.S. ${ }^{1}$, Wang J. ${ }^{3}$, Williams R. ${ }^{1}$, Unruh J.R. ${ }^{5}$, Greenberg C.H. ${ }^{2}$, Jacobs E.Y. ${ }^{3}$, Yu Z. ${ }^{8}$, de la Cruz M.J. ${ }^{8}$, Mironska R. ${ }^{1}$, Stokes D.L. ${ }^{7}$, Aitchison J.D. ${ }^{4}$, Jarrold M.F. ${ }^{6}$, Gerton J.L. ${ }^{5}$, Ludtke S.J. ${ }^{9}$, Akey C.W. ${ }^{10}$, Chait B.T. ${ }^{3}$, Sali A. ${ }^{2}$, and Rout M.P. ${ }^{1}$

1. Department of Bioengineering and Therapeutic Sciences, Department of Pharmaceutical Chemistry, and California Institute for Quantitative Biosciences, University of California, San Francisco, CA, USA

2. Laboratory of Cellular and Structural Biology, Rockefeller University, New York, NY, USA

3. Laboratory of Mass Spectrometry and Gaseous Ion Chemistry, Rockefeller University, New York, NY, USA

4. Institute for Systems Biology and Center for Infectious Disease Research, Seattle, WA, USA

5. Stowers Institute for Medical Research, Kansas City, MO, USA

6. Department of Chemistry, Indiana University, Bloomington, IN, USA

7. Skirball Institute and Department of Cell Biology, New York University School of Medicine, New York, NY, USA

8. Janelia Research Campus, Howard Hughes Medical Institute, Ashburn, VA, USA

9. National Center for Macromolecular Imaging, Verna and Marrs McLean Department of Biochemistry and Molecular Biology, Baylor College of Medicine, Houston, TX, USA

10. Department of Physiology and Biophysics, Boston University School of Medicine, Boston, MA, USA

Nuclear Pore Complexes (NPCs) are large proteinaceous assemblies studded through the nuclear envelope (NE), the double-membraned barrier surrounding the nucleus; they are the sole mediators of macromolecular transport between the nucleus and the cytoplasm, and carry key regulatory platforms for numerous nuclear processes [1]. NPCs are also major targets for viral manipulation, and defects in this transport machine are directly linked to human diseases, including cancers [2]. Each NPC is an 8-fold symmetric, cylindrical assembly consisting of $\sim 500$ copies of $\sim 30$ different proteins (nucleoporins or Nups). These Nups assemble into subcomplexes that form higher order structures called spokes. Eight spokes assemble into even larger modules: coaxial outer and inner rings form a symmetric core scaffold, which is connected to a membrane ring, a nuclear basket, and cytoplasmic RNA export complexes [3]. The scaffold surrounds a central channel, which is formed in part by multiple intrinsically disordered Phe-Gly (FG) repeat motifs extending from nucleoporins termed FG Nups. These FG motifs mediate selective nucleocytoplasmic transport through specific interactions with nuclear transport factors (NTRs) carrying their cognate macromolecular cargoes [4].

We used an integrative structure determination strategy to determine a subnanometer precision structure for the entire 552-protein, 52.3 MDa yeast NPC by satisfying diverse data including stoichiometry, a cryo-electron tomography map, and chemical cross-links. We also performed functional analysis of NPC perturbations using ODELAY [5] and heat-mapped these data to interpret the structure of the NPC in a functional context at sub-nanometer precision $[6,7]$.

We found that at the heart of the inner ring, rigid diagonal columns reinforce the NPC's structural integrity (formed by Nic96 as a keystone and flanked by Nup157 and Nup170). Membrane-binding and transmembrane Nups are strategically placed throughout the core scaffold to stabilize pore membrane 
curvature and clamp the NPC to the NE. Connectors run the length of each spoke, flexibly tying together all the major modules in the NPC. The spokes are connected by small interfaces between the adjacent Ntermini of Nup170. The NPC's architecture is reminiscent of a suspension bridge, in which rigid supporting columns are firmly anchored to a substrate while flexible suspension cables connect the columns and roadway, to provide a strong and resilient structure. We showed that most FG Nup anchor points face inward, towards the NPC central channel, to generate a highly concentrated milieu of FG repeats: FxFG/FG repeats form mRNA docking "traps" at the entrance and exit of the channel, whilst GLFG repeats help form a cytoplasmically biased permeability barrier. Whereas the core scaffold is symmetric about the plane of the NE, the two machineries associated with RNA processing and transport - the basket and export platform - are located at the nuclear and cytoplasmic faces of the NPC, respectively. At the core of the export platform is the Nup82 complex, whose coiled-coil bundle is attached to the Nup85/Seh1 arm and hub region of the Nup84 complex in the cytoplasmic outer ring. Together, they form a lateral gantry facing the central channel. Gle1 extends from the Nup82 complex by an $\alpha$-helical rod that holds itself, the RNA helicase Dbp5, and the FG repeat-carrying Nup42 over the middle of the central channel $[7,8]$. As a result, numerous transport factor docking sites and the ATPdependent RNA remodeling proteins are aligned above the cytoplasmic exit of the NPC to efficiently receive exporting RNAs to remodel and then release them into the cytoplasm.

\section{References:}

[1] Ptak, C., J.D. Aitchison, and R.W. Wozniak, Curr. Opin. Cell Biol., 28 (2014), p. 46.

[2] Simon, D.N. and M.P. Rout, 773 (2014).

[3] Alber, F. et al, Nature, 450 (2007), p. 695.

[4] Stanley, G.J., A. Fassati, and B.W. Hoogenboom, Semin Cell Dev Biol, (2017).

[5] Herricks, T. et al, G3, 7 (2017), p. 279.

[6] Fernandez-Martinez, J. et al, J. Cell Biol., 196 (2012), p. 419.

[7] Fernandez-Martinez, J. et al, Cell, 167 (2016), p. 1215-1228.e25.

[8] Knockenhauer, K.E. and T.U. Schwartz, Cell, 164 (2016), p. 1162.

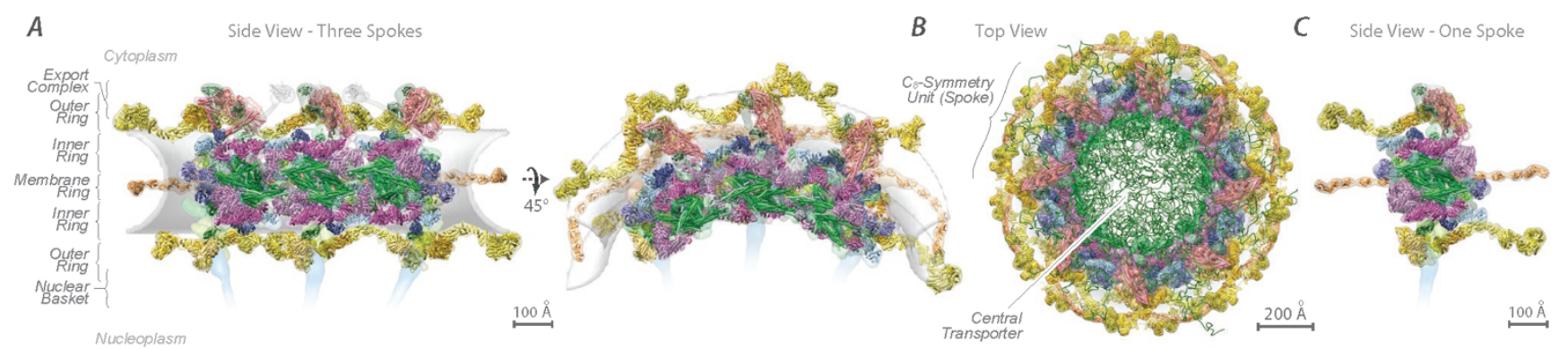

Figure 1. Structural dissection of the NPC. The complete structure of the NPC and its components. For each Nup, the localization probability density of the ensemble of structures is shown with a representative structure from the ensemble embedded within it. The structure is shown in different orientations, with a model of the pore membrane region shown in grey. (A) Two views of three consecutive NPC spokes (C8-symmetry units), showing how the coaxial outer, inner, and membrane rings run continuously between spokes. (B) Top cytoplasmic view of the complete NPC structure with modeled FG repeat regions (green). Scale bar 200 A. (C) Front view of a single NPC spoke. Scale bar 100 Å. 